Enriquecimiento

de las habilidades
Enriquecimiento de las habilidades | Revista Virtual Universidad Católica del Norte, 64, 65-91

ISSN: 0124-5821 (En línea)

González Arreola, M. R., y Chávez Soto, B. I. (septiembre-diciembre, 2021). Enriquecimiento de las habilidades cognitivas de niños con aptitud sobresaliente. Revista Virtual Universidad

Católica del Norte, (64), 65-91. https://www.doi.org/10.35575/rvucn.n64a4

\title{
Enriquecimiento de las habilidades cognitivas de niños con aptitud sobresaliente
}

Strategies to enrich cognitive skills of children with outstanding intelligence

\author{
Mercedes Rosalía González Arreola \\ Maestra en Psicología \\ Facultad de Estudios Superiores Zaragoza, Universidad Nacional Autónoma de México \\ Sinaloa, México \\ meroga_agorem@hotmail.com
}

Orcid: https://orcid.org/0000-0001-8811-4974

\section{Blanca Ivet Chávez Soto}

Doctora en Psicología Educativa y del Desarrollo

Facultad de Estudios Superiores Zaragoza, Universidad Nacional Autónoma de México

Ciudad de México, México

mil_chavez@hotmail.com

Orcid: https://orcid.org/0000-0001-5922-2351

Recibido: 2 de julio de 2020

Evaluado: 30 de noviembre de 2020

Aprobado: 6 de abril de 2021

Tipo de artículo: Investigación Científica y Tecnológica

\section{Resumen}

De acuerdo con investigaciones recientes, los programas de enriquecimiento pueden ser una alternativa pedagógica que permite estimular las habilidades cognoscitivas de los estudiantes con necesidades educativas especiales. A partir de estas ideas, se planteó como objetivo diseñar e instrumentar el programa de enriquecimiento Meccano para favorecer las habilidades cognitivas de niños con aptitud sobresaliente. El presente es un estudio mixto, con un diseño Pre test-Post test, donde participaron seis niños con aptitud sobresaliente, quienes obtuvieron puntuaciones 
Enriquecimiento

de las habilidades
Enriquecimiento de las habilidades | Revista Virtual

Universidad Católica del Norte, 64, 65-91

ISSN: 0124-5821 (En línea)

iguales o superiores al percentil 75, en por lo menos tres de las siguientes variables: creatividad, razonamiento perceptual, compromiso con la tarea, autoconcepto académico y nominación del maestro. El perfil de los alumnos se complementó con la prueba WISC IV, y con base en la información se diseñó el Programa de enriquecimiento Meccano, a partir de las propuestas de Duarte y Feuerstein. Después del análisis cuantitativo y cualitativo, en el Post test, se observaron incrementos significativos en las variables de creatividad e inteligencia, por lo que se recomienda continuar con el proyecto para fortalecer las habilidades superiores y lograr que los niños tengan un desarrollo óptimo en los distintos ámbitos de su vida.

Palabras Clave: Creatividad; Habilidades del pensamiento; Inteligencia.

\begin{abstract}
According to recent research, enrichment programs can be a pedagogical alternative that stimulates the cognitive skills of students with special educational needs. Based on these ideas, the objective was to design and implement the Meccano enrichment program to stimulate the cognitive skills of children with outstanding intelligence. This is a mixed research, with a Pre test-Post test design where six children with outstanding intelligence participated, who obtained scores equal to or greater than the 75 th percentile in at least three of the following variables: creativity, perceptual reasoning, commitment to homework, academic self-concept and teacher nomination. The students' profile was complemented with the WISC IV test and based on the information, the Meccano Enrichment Program was designed based on the proposals of Duarte and Feuerstein. After the quantitative and qualitative analysis in the Post test, significant increases were observed in the variables of creativity and intelligence, so it is recommended to continue with the project to strengthen superior skills and ensure that children have optimal development in different areas of their life.
\end{abstract}

Keywords: Creativity; Cognitive abilities; Intelligence. 
Enriquecimiento

de las habilidades
Enriquecimiento de las habilidades | Revista Virtual

Universidad Católica del Norte, 64, 65-91

ISSN: 0124-5821 (En línea)

\section{Introducción}

La educación se consolidó como un derecho universal y obligatorio en Europa, después de la Segunda Guerra Mundial; a partir de ahí se realizaron acciones para ofrecer atención a todas las personas (Frola, 2004). Dichas acciones influyeron en las políticas educativas de México, y un hecho relevante se dio en 1970 con la creación de la Dirección General de Educación Especial, cuya finalidad fue organizar, dirigir, desarrollar, administrar y vigilar el sistema federal de Educación Especial (EE), y desde ese momento se han realizado diversos convenios internacionales para promover la atención educativa de las personas que presentan necesidades educativas especiales (NEE).

Los cambios establecidos en las políticas han contribuido a impulsar que se tengan mejores condiciones de vida y de desarrollo, además de promover ambientes en la sociedad con mayores niveles de competitividad (Rueda Romero, 2016). Una de estas cosas es la creación de la $\mathrm{EE}$, que se definió como "aquella atención especializada dirigida a niños quienes por diversas razones no pueden obtener un aprendizaje satisfactorio con el currículo escolar tal como se proporciona normalmente" (Williams, 2001).

En México, durante 1992 se produjeron grandes transformaciones en la estructura de la EE, debido a la modificación del artículo 41 de la Ley General de Educación. El país inició formalmente el proceso de integración educativa, y se transformó el modelo médico por un modelo social-educativo (Diario Oficial de la Federación, 2012, como se citó en Romero y García, 2013). Dicho artículo estipuló que:

La educación especial está destinada a individuos con discapacidades transitorias o definitivas, así como aquellos con aptitudes sobresalientes. Atenderá a los educandos de manera adecuada a sus propias condiciones con equidad social.

(...) esta educación propiciará su integración a los planteles de educación regular mediante la aplicación de métodos, técnicas y materiales específicos.

Esta educación incluye orientación a los padres o tutores, así como también a los maestros y personal de escuelas de educación básica regular que integren alumnos con necesidades especiales de educación. (Secretaría de Educación Pública, 2006, p. 15) 
El Programa Nacional de Educación (PRONAE 2001-2006) reconoció que la población que presentaba alguna discapacidad era uno de los principales grupos en situación de vulnerabilidad, respecto a su acceso, permanencia y egreso del Sistema Educativo Nacional, y estableció los lineamientos para la atención de aquellos con aptitudes sobresalientes (Secretaría de Educación Pública -SEP-, 2006, pp. 1215). Ante estas políticas en la Ciudad de México se creó el Programa de Niños y Niñas Talento y el Programa de Potencialidades (Sistema para el Desarrollo Integral de la Familia del Distrito Federal, 2014), con el objetivo de apoyar a estos alumnos para desarrollar y perfeccionar sus habilidades de forma integral

Por otro lado, en el Foro Mundial sobre la Educación en la República de Corea (2015), se aprobó la Declaración de Incheon, en la cual se reconoció la importancia de garantizar una enseñanza inclusiva, equitativa y de calidad, para promover oportunidades de aprendizaje durante toda la vida, para fomentar la creatividad, conocimiento, valores, competencias básicas de lectura, escritura, cálculo, aptitudes analíticas y de solución de problemas (Unesco y Unicef, 2015).

De esta manera, para contribuir con las demandas en materia educativa de la población sobresaliente, se desarrolló la presente investigación, la cual, a continuación, ostenta una propuesta de atención para niños de educación primaria, ubicados en esta categoría; en tanto, si bien sería complicado particularizar las características de cada uno de los alumnos, lo que sí se puede hacer es "buscar aquellas características básicas de su proceso de aprendizaje que han de aportar criterios y planteamientos globales que necesariamente han de concretarse e individualizarse en cada alumno y en cada situación de aprendizaje" (De Pablo-Blanco y Rodríguez, 2010).

\section{Marco teórico}

Se han empleado muchos conceptos para referirse a los niños con aptitud sobresaliente. En la literatura se considera que el primer término usado para esta población fue propuesto por Galton (Covarrubias, 2018; Sánchez, 2003), quien utilizó la palabra "Genio" para definir a las personas que poseen un alto grado de inteligencia; además, este autor comenzó con el estudio de las personas poseedoras de cualidades y capacidades de alto nivel, por medio del análisis estadístico de habilidades intelectuales, como el razonamiento matemático, verbal, espacial y abstracto.

Años más tarde, Binet (como se citó en Chávez, 2008) desarrolló un instrumento de evaluación para detectar problemas de aprendizaje, el cual se convirtió en uno de los más 
conocidos y empleados para medir el coeficiente intelectual (CI). Esta escala fue adaptada por Terman a la realidad estadounidense y surgió la Prueba de Inteligencia Stanford-Binet (Covarrubias, 2018; López, 2008), que sirvió para hacer el primer estudio longitudinal con estudiantes superdotados, que son aquellas personas que presentan un nivel intelectual superior a 130 (Chávez, 2008).

Actualmente, en México, se emplea el término aptitud sobresaliente para referirse a aquellos alumnos que destacan en el campo científico, tecnológico, humanístico-social, artístico o motriz, y son atendidos dentro de la educación especial, debido a que sus aptitudes no siempre se encuentran vinculadas con los contenidos escolares (SEP, 2006). Es pertinente reconocer que en el país se han dado avances en torno a la categoría de los estudiantes sobresalientes, pero aún existen retos por superar, ya que menos del $1 \%$ de estos niños es identificado y recibe la atención que se desea para favorecer su potencial (Chávez, 2014).

Existen diversos modelos para detectar a los estudiantes sobresalientes y las propuestas actuales incluyen variables como la inteligencia, creatividad, motivación, y autoconcepto; también, se ha considerado la participación de los profesores y la familia para complementar la evaluación. Con base en lo anterior, se creó la propuesta multidimensional para detectar alumnos sobresalientes en escuelas de educación básica de la Ciudad de México, la cual ha demostrado una efectividad de $83 \%$ para seleccionar a los niños ubicados en la categoría (Chávez, 2014; Chávez y Acle, 2018; Chávez et al., 2009. Adicionalmente, a los seleccionados se les aplica la prueba WISC IV para complementar el perfil, el cual sirve para la creación de los programas de intervención educativa (Marchesi et al., 2005; Pérez et al., 2015).

Para Booij et al. (2016), Chan et al. (2020), Chen \& Chen (2020), García-Perales y Almeida (2019), Golle et al. (2019), y Sánchez (2003), el enriquecimiento es una alternativa eficaz en la atención de los niños sobresalientes, debido a que amplía la visión del alumno, al favorecer habilidades específicas y desarrollar nuevas destrezas; el aprendizaje de alguna materia o tema se fortalece con más profundidad gracias a la innovación y creatividad, adicionales a las establecidas en el currículo. Cabe mencionar que el ser creativo es una habilidad que ayuda a desarrollar asociaciones, establecer relaciones y combinaciones, e integrar ideas con conceptos diferentes, lo cual es indispensable para la época actual (Hernández, et al., 2015). 
Enriquecimiento

de las habilidades
Enriquecimiento de las habilidades | Revista Virtual

Universidad Católica del Norte, 64, 65-91

ISSN: 0124-5821 (En línea)

El Programa de enriquecimiento Instrumental se diseñó sobre la teoría de la modificabilidad estructural cognitiva de Feuerstein (como se citó en Velarde, 2008), quien indicó que el bajo rendimiento escolar es producto del uso ineficaz de las funciones, que son los prerrequisitos para aprender. Los objetivos específicos de la propuesta fueron: 1) corregir las funciones deficientes, 2) adquirir conceptos básicos, vocabulario y operaciones, 3) desarrollar la motivación intrínseca, 4) crear cierto nivel de insight o pensamiento reflexivo, 5) Desplegar y facilitar la auto-percepción del individuo, y 6) formar hábitos de trabajo. Además, se interesó en trabajar aspectos motivacionales y metacognitivos con tareas aplicables, tanto en el ámbito educativo como en la vida cotidiana.

Feuerstein (1980) ha señalado que algunas habilidades cognitivas que desarrolla el Programa de enriquecimiento Instrumental son: la capacidad de percibir los datos con precisión y exactitud; establecer relaciones entre objetos, acontecimientos e ideas; comparar, clasificar y organizar la información; diferenciar, analizar e integrar; trabajar de manera planificada; utilizar el lenguaje con claridad y precisión, comprender, dar instrucciones y argumentar; identificar los problemas y sus causas; pensar antes de actuar, explorar alternativas y prever consecuencias, por lo que en estas estrategias se ha buscado que los individuos sean los protagonistas de su propio proceso de aprendizaje, para que los aprendizajes perduren en el tiempo (Howie, 2015; Mateus, 2017), y que ayudan a favorecer el pensamiento crítico, la comunicación y la reflexión que son indispensables para el aprendizaje (Montoya y Monsalve, 2008).

Algunas de las habilidades propuestas por Feuerstein (Howie, 2015; Mateus, 2017; Ushiñahua y Vílchez, 2016), y que se retomaron para la investigación son: Observación (percepción de estímulos de manera parcial o global, reconocer detalles de forma precisa, conservar la constancia de los objetos, cuando tienen variaciones en alguno de sus atributos: tamaño, forma, cantidad, dirección), organización de puntos (proyección de relaciones virtuales a través del reconocimiento de figuras escondidas en una secuencia numérica, identificar y dibujar las formas dadas dentro de la nube de puntos), orientación espacial (sistema de referencia estable -Norte-Sur-Este-Oeste- y relativo -Frente-Atrás- Arriba-Abajo- para describir las relaciones espaciales), comparación (repertorio de atributos para comparar los estímulos, diferenciar entre elementos por medio de parámetros), clasificación (reunir información y organizarla de manera más eficiente, al determinar el universo al cual pertenecen los elementos 
Enriquecimiento

de las habilidades
Enriquecimiento de las habilidades | Revista Virtual

Universidad Católica del Norte, 64, 65-91

ISSN: 0124-5821 (En línea)

dados para agruparlos en una categoría) y percepción analítica (estrategias para la integración, síntesis de las partes de un todo, de acuerdo con ciertos objetivos).

Con base en lo antes descrito, en la presente investigación se consideró pertinente retomar las propuestas de Tzuriel y Feuerstein (1992) y de Duarte (2001); la primera, se ha empleado principalmente en población que presenta bajos niveles cognitivos, y la segunda es una alternativa para favorecer la creatividad (Bahar \& Ozturk, 2018; Klimenko y Botero, 2016). Se hicieron adaptaciones de acuerdo con el perfil de los estudiantes sobresalientes, y en este estudio se presentan los hallazgos de esta alternativa educativa. De ahí que el objetivo de la presente investigación fue diseñar e instrumentar el programa de enriquecimiento Meccano para favorecer las habilidades cognitivas de niños con aptitud sobresaliente.

\section{Metodología}

Para la recolección y análisis de datos se emplearon técnicas cualitativas y cuantitativas, por ello el estudio se categorizó como tipo mixto, con un diseño Pre test-Post test (Creswell \& Plano, 2007), el cual hace posible determinar si hay cambios favorables o no, y hacer un análisis comparativo del antes y después del programa de intervención de enriquecimiento "Proyecto Meccano". Por otra parte, el estudio es tipo cuasi- experimental con un solo grupo con Pretest y Post test, por esta razón no se requirió un grupo control (Kerliger y Lee, 2001).

\section{Participantes}

En la primera etapa (Pre test) de esta investigación participaron 83 alumnos (40 mujeres y 43 hombres), con un rango de edad de siete a ocho años (Medad= 7.78, D.E.=0.415), inscritos en tercer grado de educación primaria, que asistían en el turno matutino, así como tres profesoras de cada uno de los grupos: A, B y C.

Obtenidos los resultados de esa primera etapa, se realizó una reunión con los padres de familia, donde se les ofreció una explicación de las pruebas realizadas, se les entregaron los puntajes de cada uno de los alumnos y, en seguida, se les extendió una invitación para que sus hijos fueran partícipes en el desarrollo de las actividades de "Proyecto Meccano". Sólo seis de los padres firmaron el consentimiento informado y aceptaron participar. 
Enriquecimiento

de las habilidades
Enriquecimiento de las habilidades | Revista Virtual

Universidad Católica del Norte, 64, 65-91

ISSN: 0124-5821 (En línea)

Partiendo de lo anterior, para la Fase II (diseño e instrumentación del Proyecto Meccano) y Fase III Post test (evaluación final), se trabajó con seis alumnos (dos mujeres y cuatro hombres) con aptitud sobresaliente, con un rango de edad de siete a ocho años $(\mathrm{Medad}=7.75, \mathrm{DE}=0.463)$, inscritos en tercer grado de educación primaria pública de la Ciudad de México.

\section{Instrumentos}

Prueba de Pensamiento Creativo Forma Figural A, de Torrance (2008): evalúa las producciones creativas a través de tres actividades: componer un dibujo, acabar un dibujo y líneas paralelas. Los indicadores que valora son: fluidez, originalidad, elaboración, cierre y títulos. Tiene un índice de confiabilidad de 0.90 (Zacatelco y Chávez, 2015).

Test de Matrices Progresivas de Raven Forma Coloreada (Raven et al., 1993): Mide la capacidad intelectual del niño, a través de 36 problemas agrupados en tres series: A, AB y B, con seis opciones de respuesta, una correcta y cinco erróneas. Tiene una confiabilidad de 0.774 , obtenida por un Alpha de Cronbach (Chávez, 2014).

Escala de Compromiso con la Tarea (Zacatelco, 2005): Conformada por 18 reactivos con opción de respuesta tipo Likert con seis intervalos. Evalúa la motivación intrínseca a través de los factores de interés, persistencia y esfuerzo en actividades escolares y extracurriculares. Su índice de confiabilidad fue de 0.79 , obtenido por un Alpha de Cronbach.

Escala de Autoconcepto Académico (Chávez, 2014): Conformada por 31 afirmaciones que describen qué tan hábil se siente el alumno en sus materias escolares; se responde con una escala tipo Likert de siete opciones. Su índice de confiabilidad es de 0.848, obtenida por un Alpha de Cronbach.

Escala de Nominación del Maestro (Zacatelco et al., 2013): Consta de 37 ítems que ayudan al docente a identificar alumnos con aptitud sobresaliente, a través de cuatro dimensiones: habilidades cognoscitivas, creatividad, compromiso con la tarea y aspectos socioafectivos. Su índice de confiabilidad es de 0.934 .

Escala Wechsler de Inteligencia para Niños WISC-IV (Wechsler, 2005): Evalúa la capacidad intelectual, a través de cuatro escalas: Comprensión Verbal, Razonamiento Perceptivo, Memoria de Trabajo y Velocidad de Procesamiento, con las cuales se obtiene un C.I. total. 
Enriquecimiento

de las habilidades
Enriquecimiento de las habilidades | Revista Virtual

Universidad Católica del Norte, 64, 65-91

ISSN: 0124-5821 (En línea)

Proyecto Meccano: Se diseñaron 92 actividades para favorecer los procesos cognitivos de observación, organización de puntos, orientación espacial, comparación, clasificación y percepción analítica.

\section{Procedimiento}

El trabajo se organizó en tres fases:

Fase I Pre-test (Detección de los niños sobresalientes): Se platicó con la directora de la primaria para realizar la identificación de los niños; además, los padres firmaron el consentimiento informado. Durante dos semanas se aplicaron los instrumentos: Prueba de Pensamiento Creativo, Test de Matrices Progresivas de Raven, Escala de Compromiso con la Tarea y Escala de Autoconcepto Académico, a un total de 83 alumnos (40 mujeres y 43 hombres), inscritos en tercer grado de educación primaria. También, participaron dos docentes, los cuales llenaron la lista de nominación. Los datos se capturaron en el programa estadístico SPSS V22, y se obtuvieron los estadísticos descriptivos y el percentil 75. Con base en dicha estimación, se seleccionó a los estudiantes que obtuvieron puntuaciones iguales o superiores a este percentil, en tres de los cinco instrumentos (Chávez, 2014; Mönks, 1999; Renzulli, 2011). Posteriormente, a los niños seleccionados, y cuyos padres aceptaron que participaran en el Proyecto Meccano, se les aplicó la prueba WISC IV. Los resultados permitieron reconocer, como principales fortalezas en los niños, altos niveles de creatividad, inteligencia general, autoconcepto, compromiso con la tarea; se observaron elevadas habilidades para analizar las relaciones entre los estímulos visuales, seguir estrategias y diseñar un plan de trabajo para resolver un problema.

Por otro lado, el perfil de los niños permitió reconocer que las áreas a favorecer eran las capacidades para percibir los estímulos, de manera parcial y global, fomentar el trabajo cooperativo, fortalecer la autoconfianza, desarrollar la habilidad de recuperar información, memorización, comprensión verbal, e incrementar el repertorio de atributos para comparar los estímulos y encontrar diferencias, así como reunir información y clasificarla.

Fase II (Diseño e instrumentación del Proyecto Meccano): Con los datos de la Fase I se diseñó el Proyecto Meccano con un total de 92 actividades, las cuales se realizaron en 35 
Enriquecimiento

de las habilidades
Enriquecimiento de las habilidades | Revista Virtual

Universidad Católica del Norte, 64, 65-91

ISSN: 0124-5821 (En línea)

sesiones, con una duración aproximada de 90 minutos, dos veces por semana, organizadas como se indica en la tabla 1. Se inició el trabajo con la habilidad de observación; terminadas las cinco sesiones, se procedió a trabajar con organización de puntos, durante cuatro sesiones; después, orientación espacial, en cinco, seguida de comparación, en siete; más tarde, la habilidad de clasificación, durante cinco sesiones; y finalmente, la habilidad de percepción analítica, en nueve sesiones.

\section{Tabla 1}

Proyecto Meccano por sesiones y actividades.

\begin{tabular}{lcc}
\hline Habilidad del pensamiento & Número de Sesiones & Número de Actividades \\
\hline Observación & Cinco & 16 \\
Organización de puntos & Cuatro & 20 \\
Orientación especial & Cinco & 10 \\
Comparación & Siete & 18 \\
Clasificación & Cinco & 15 \\
Percepción analítica & Nueve & 13 \\
\hline
\end{tabular}

Nota: Elaboración propia.

Los objetivos puntuales en esta fase fueron: i) favorecer la habilidad de percibir los estímulos, no sólo de manera parcial o globalmente. Es decir, buscar que reconozcan detalles de forma precisa; ii) fomentar la capacidad para conservar la constancia de los objetos, cuando estos experimentan variaciones en alguno de sus atributos (tamaño, forma, cantidad, dirección); iii) ejercitar la función de proyección de relaciones virtuales, a través del reconocimiento de figuras escondidas en una secuencia numérica; iv) identificar y dibujar las formas dadas dentro de una nube de puntos; v) apropiarse de un sistema de referencia estable (Norte-Sur-Este-Oeste), aunque relativo (Frente-Atrás- Arriba-Abajo), para describir las relaciones espaciales; y vi) comprender el vocabulario empleado en cada uno de los módulos de Organización de puntos y Orientación espacial, el cual es necesario para realizar las actividades.

Se elaboró un portafolio de evidencias con los productos desarrollados por los participantes durante la instrumentación del programa, los cuales se evaluaron en tres momentos (King y Cambell-Allan, 2006): 
Enriquecimiento

de las habilidades
Enriquecimiento de las habilidades | Revista Virtual

Universidad Católica del Norte, 64, 65-91

ISSN: 0124-5821 (En línea)

Inicial: los niños realizaron ejercicios con el propósito de valorar su capacidad en las áreas que se favorecerían mediante el programa.

Intervención: se diseñaron estrategias para desarrollar las capacidades cognoscitivas de los estudiantes.

Evaluación final: se realizaron actividades semejantes a las efectuadas al inicio de cada bloque, con el propósito de conocer las diferencias derivadas del "Proyecto Meccano". Para conocer los cambios en los niños se retomaron los aciertos de los ejercicios de la evaluación inicial y final.

Fase III Post test (Evaluación final): Al término del programa, a los niños se les aplicaron los cuatro instrumentos empleados durante el Pre-test, con el propósito de reconocer los efectos del programa.

\section{Resultados}

Los resultados se presentan en dos partes; la primera, corresponde a los cambios observados durante la instrumentación del programa; en la segunda, se muestra la evaluación Pretest- Post test.

\section{Resultados de los niños durante la aplicación del Proyecto Mecanno}

Se empleó la prueba no paramétrica de Wilcoxon para comparar las puntuaciones obtenidas en la evaluación inicial y final de cada módulo de las habilidades del pensamiento, y conocer los cambios en los alumnos. Cabe señalar que los resultados reportaron incrementos significativos en las seis capacidades que se trabajaron: Observación $(Z=0.027, p<0.05)$, Organización $(Z=0.028, p<0.05)$, Orientación espacial $(Z=0.46, p<0.05)$, Comparación $(Z=$ $0.46, p<0.05)$, Clasificación $(Z=0.026, p<0.05)$ y Percepción analítica $(Z=0.027, p<0.05)$, tal y como se observa en la figura 1. 
Enriquecimiento

de las habilidades
Enriquecimiento de las habilidades | Revista Virtual

Universidad Católica del Norte, 64, 65-91

ISSN: 0124-5821 (En línea)

\section{Figura 1}

Puntuaciones medias obtenidas por los alumnos con aptitud sobresaliente, en la evaluación inicial y final de cada módulo de las habilidades del pensamiento

250
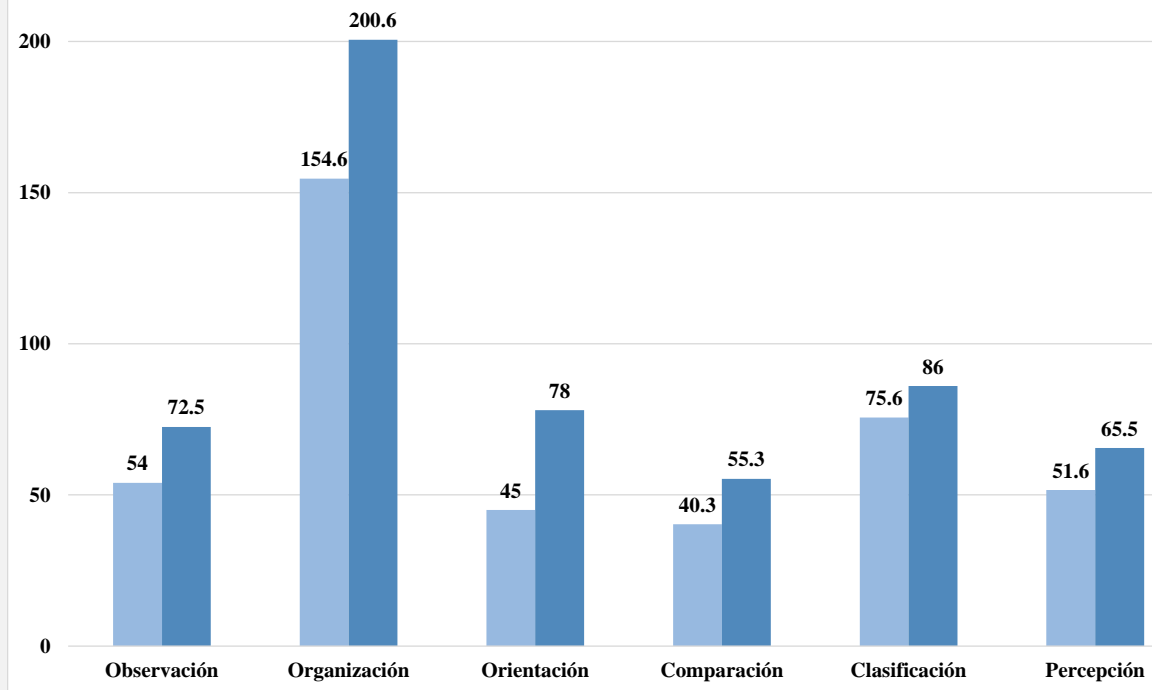

Pre Tets $\quad$ Post Test

Nota: Elaboración propia.

Con el aumento de las medias y el análisis de los productos del portafolio de evidencia se observó que los niños percibieron los estímulos de manera parcial y global, y reconocieron detalles de forma precisa; conservaron la constancia de los objetos, cuando estos experimentaron variaciones en alguno de sus atributos; comprendieron el vocabulario empleado, necesario para realizar las actividades. Así mismo, incrementaron el repertorio de atributos, por los cuales se pueden comparar los estímulos; diferenciaron elementos por medio de parámetros; y lograron reunir información y organizarla de manera más eficiente.

\section{Resultados Pretest - Post test}

Las puntuaciones de los estudiantes de cada uno de los instrumentos en el Pre y Post test se analizaron con la prueba no paramétrica de Wilcoxon, y se encontraron incrementos 


\section{Enriquecimiento}

de las habilidades
Enriquecimiento de las habilidades | Revista Virtual

Universidad Católica del Norte, 64, 65-91

ISSN: 0124-5821 (En línea)

significativos en la inteligencia evaluada con Raven $(Z=0.028, \mathrm{p}<0.05)$ y en la creatividad $(Z=$ $0.046, \mathrm{p}<0.05)$. En cuanto al compromiso con la tarea $(P=0.833$, $\mathrm{a}<0.05)$ y autoconcepto $(\mathrm{P}=$ $0.116, \mathrm{a}<0.05$ ) no se observaron aumentos significativos (ver figura 2 ).

\section{Figura 2}

Puntuaciones medias obtenidas por los alumnos con aptitud sobresaliente, en Pre-Post test de las escalas de inteligencia, creatividad, compromiso con la tarea y autoconcepto

200

180

160

140

120

100

60

40

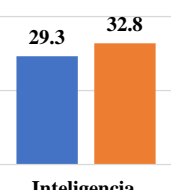

Inteligencia

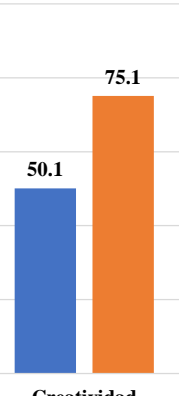

Creatividad

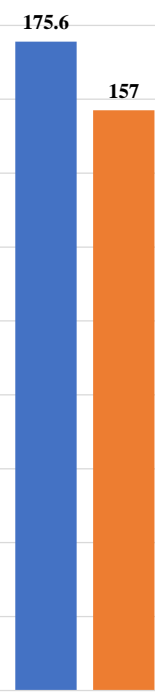

Autoconcepto

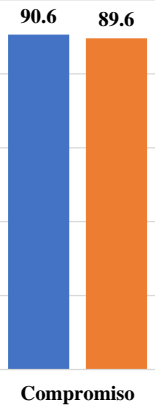

- Pre Test $\quad$ Post Test

Nota: Elaboración propia.

Para analizar los incrementos por cada alumno, en la tabla 2 se presentan los resultados. Se observó que la variable de inteligencia aumentó en los seis estudiantes y las puntuaciones están muy cerca de la máxima, que es 36 puntos. Por otra parte, cinco de los estudiantes elevaron su creatividad. Tres subieron el nivel de compromiso con la tarea y dos mejoraron el autoconcepto académico. 


\section{Enriquecimiento}

de las habilidades
Enriquecimiento de las habilidades | Revista Virtual

Universidad Católica del Norte, 64, 65-91

ISSN: 0124-5821 (En línea)

\section{Tabla 2}

Puntuaciones obtenidas por cada alumno en las pruebas Pre-Post test de Raven, creatividad, compromiso y autoconcepto.

\begin{tabular}{ccccccccc}
\hline \multirow{2}{*}{ Alumnos } & \multicolumn{2}{c}{ Raven } & \multicolumn{2}{c}{ Creatividad } & \multicolumn{2}{c}{ Compromiso } & \multicolumn{2}{c}{ Autoconcepto } \\
\cline { 2 - 9 } & Pre & Post & Pre & Post & Pre & Post & Pre & Post \\
\hline DG & 30 & $\mathbf{3 5}$ & 54 & $\mathbf{8 2}$ & 97 & $\mathbf{9 8}$ & 195 & 175 \\
\hline JI & 26 & $\mathbf{3 2}$ & 33 & $\mathbf{8 1}$ & 97 & 90 & 180 & 163 \\
\hline HR & 31 & $\mathbf{3 2}$ & 47 & $\mathbf{7 4}$ & 98 & 86 & 184 & 148 \\
\hline DL & 31 & $\mathbf{3 3}$ & 63 & 52 & 56 & $\mathbf{6 3}$ & 132 & $\mathbf{1 3 8}$ \\
\hline KB & 30 & $\mathbf{3 3}$ & 60 & $\mathbf{8 8}$ & 95 & $\mathbf{1 0 5}$ & 206 & 154 \\
\hline SJ & 28 & $\mathbf{3 2}$ & 44 & $\mathbf{7 4}$ & 101 & 96 & 157 & $\mathbf{1 6 4}$ \\
\hline
\end{tabular}

Nota: Elaboración propia. Los números resaltados en negrita son aquellos que elevaron su puntuación en la etapa Post- test.

$\mathrm{Al}$ hacer un análisis detallado sobre los cambios en la creatividad de los niños con aptitud sobresaliente, se encontró un incremento significativo en el indicador de Cierre $\mathrm{P}=0.041$, a< 0.05). En el caso de Fluidez $(\mathrm{P}=0.344, \mathrm{a}<0.05)$, Originalidad $(\mathrm{P}=0.141, \mathrm{a}<0.05)$, Elaboración $(\mathrm{P}=0.058, \mathrm{a}<0.05)$ y Título $(\mathrm{P}=0.273, \mathrm{a}<0.05)$ no presentaron diferencias significativas (ver figura 3 ). 


\section{Enriquecimiento}

de las habilidades
Enriquecimiento de las habilidades | Revista Virtual

Universidad Católica del Norte, 64, 65-91

ISSN: 0124-5821 (En línea)

\section{Figura 3}

Puntuaciones medias obtenidas por los alumnos con aptitud sobresaliente, en cada uno de los indicadores de Creatividad según Torrance (2008)

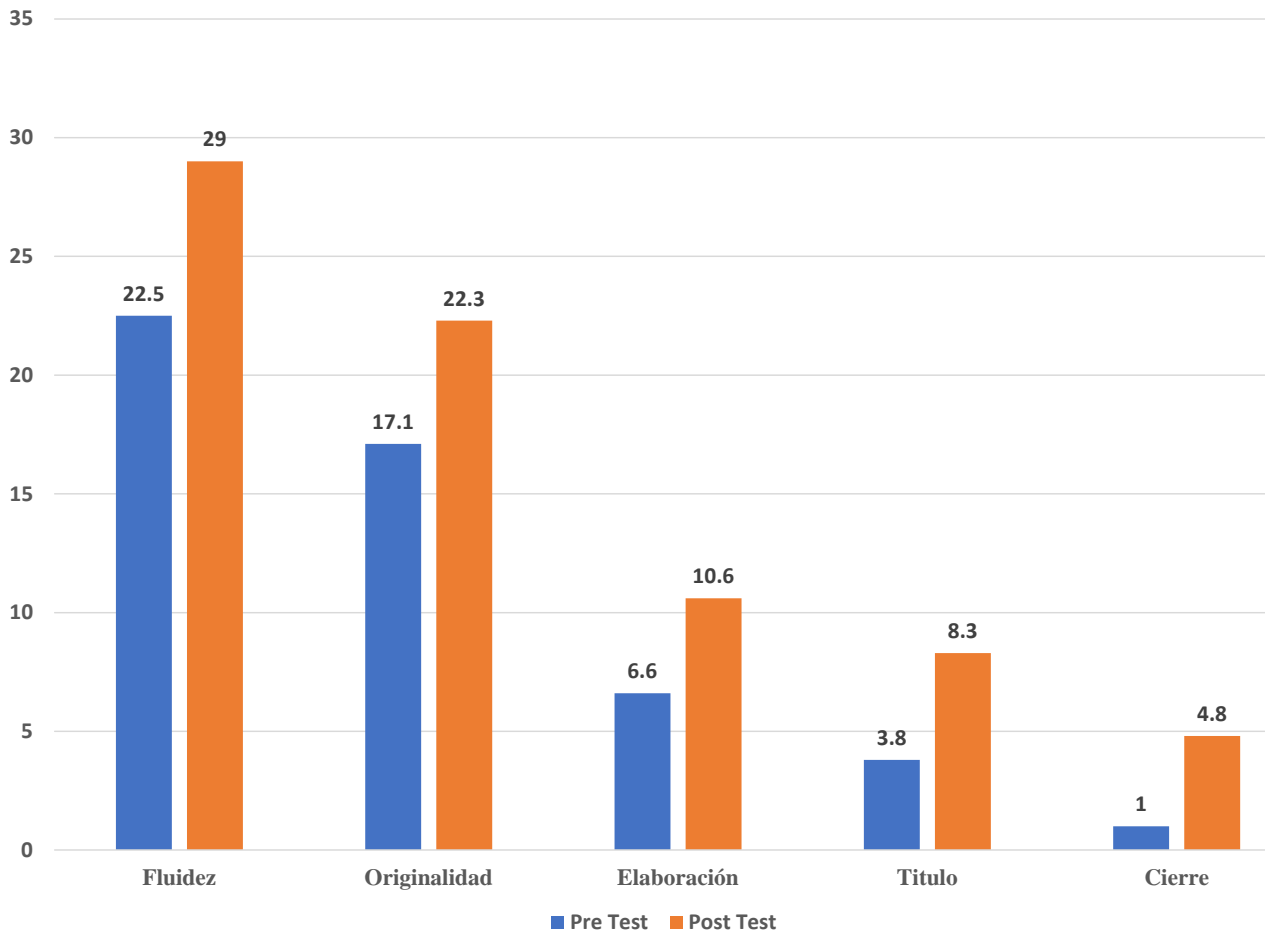

Nota: Elaboración propia.

Para comprender mejor estos cambios, se presentan dos dibujos en la figura 4, realizados por un alumno en la etapa Pre y Post test; al compararse se observó que la primera creación titulada "Un ojo" (izquierda), tuvo menos detalles y colores, respecto a la segunda (derecha), donde se colocaron más elementos dentro y fuera del estímulo. El primer título fue genérico, mientras que en el siguiente añadió una característica, la palabra "caliente", la cual coincide con el humo que dibujó, para dar a entender que el contenido de la taza es ardiente. Así mismo, el haber anexado el sol puede ser un indicador de tiempo, en este caso la mañana, ya que usualmente al desayunar se bebe una taza de café. 


\section{Enriquecimiento}

de las habilidades
Enriquecimiento de las habilidades | Revista Virtual Universidad Católica del Norte, 64, 65-91 ISSN: 0124-5821 (En línea)

\section{Figura 4}

Dibujos creados por el alumno JI la Fase Pre y Post test.
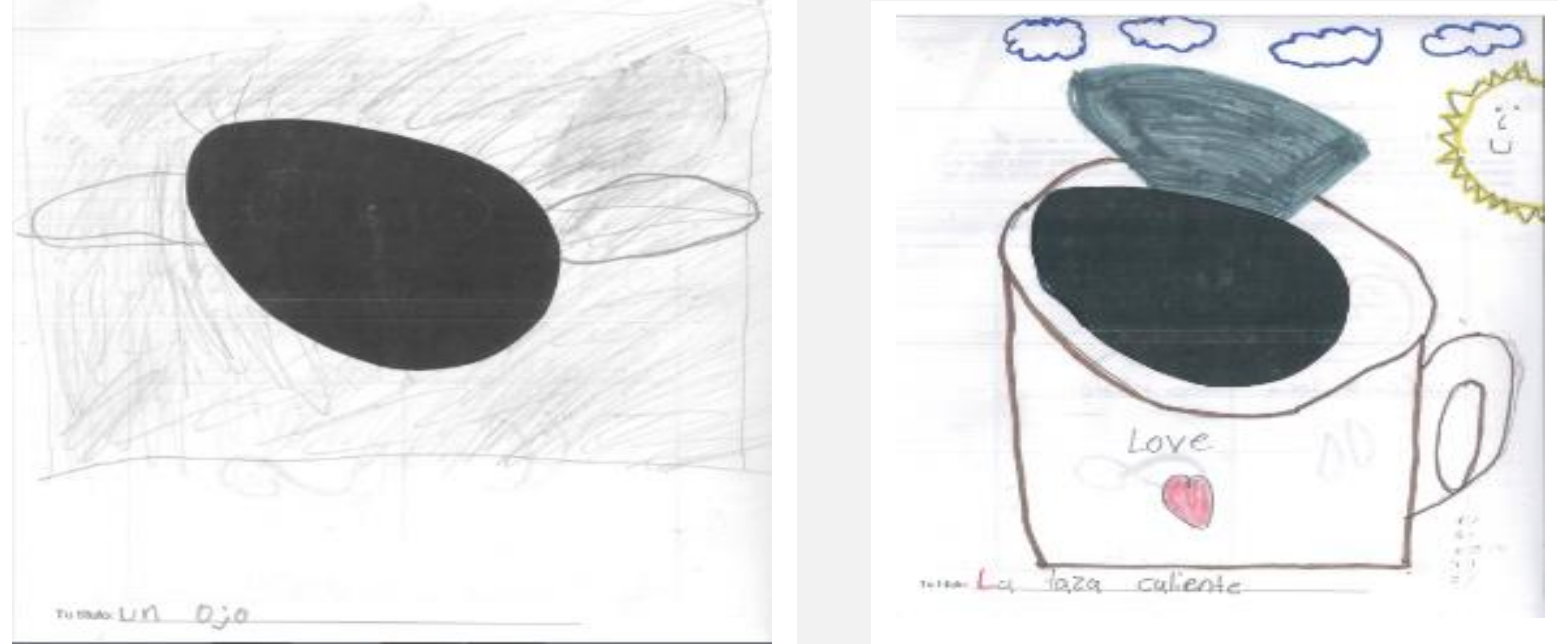

Nota: Elaboración propia.

Con respecto a la prueba WISC IV, se observó un incremento significativo en los índices de Comprensión Verbal $(\mathrm{P}=0.028, \mathrm{a}<0.05)$, Velocidad de Procesamiento $(\mathrm{P}=0.027, \mathrm{a}<0.05) \mathrm{y}$ en el CI total $(Z=0.027, p<0.05)$. En cuanto a Razonamiento Perceptual $(P=0.075, a<0.05)$ y Memoria de Trabajo $(\mathrm{P}=0.138$, $\mathrm{a}<0.05)$ hubo incrementos, pero estos no fueron estadísticamente significativos, como se observa en la figura 5. 


\section{Enriquecimiento}

de las habilidades
Enriquecimiento de las habilidades | Revista Virtual

Universidad Católica del Norte, 64, 65-91

ISSN: 0124-5821 (En línea)

\section{Figura 5}

Puntuaciones medias obtenidas por los alumnos con aptitud sobresaliente, en cada uno de los dominios de inteligencia en el Pre- Post test de WISC-IV

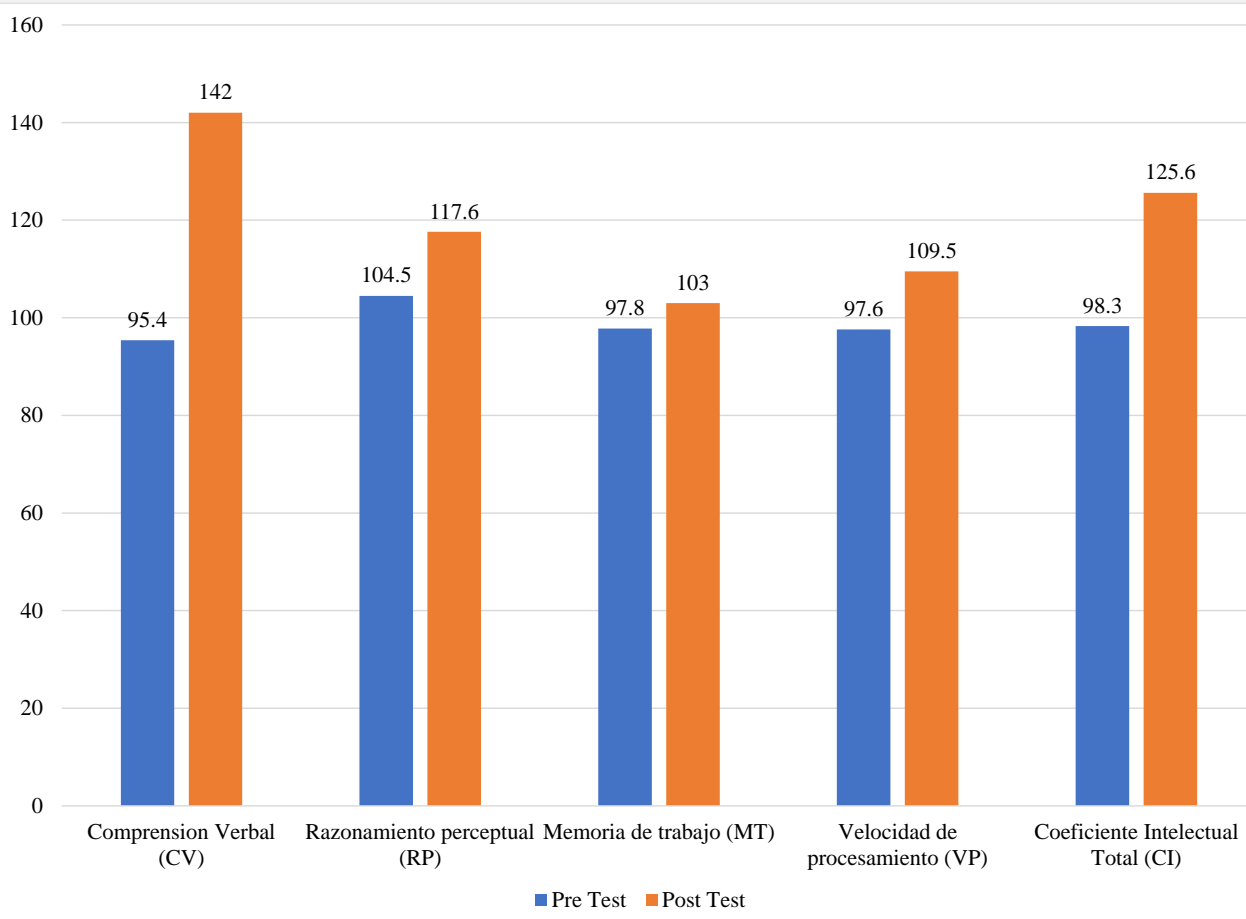

Nota: Elaboración propia.

En la Tabla 3, por su parte, se encuentran las puntuaciones obtenidas por cada alumno en el WISC-IV. Se observó que, en el área de Comprensión Verbal y Velocidad de Procesamiento, todos los niños aumentaron su calificación, mientras que, en Razonamiento Perceptual y Memoria de Trabajo, cinco lograron subir. Cabe señalar que los seis estudiantes mejoraron su CI total al finalizar el programa de intervención educativa. 
Enriquecimiento

de las habilidades
Enriquecimiento de las habilidades | Revista Virtual

Universidad Católica del Norte, 64, 65-91

ISSN: 0124-5821 (En línea)

Tabla 3

Puntuaciones obtenidas por cada alumno con aptitud sobresaliente en los dominios de inteligencia en el Pre-Post test de WISC-IV

\begin{tabular}{ccccccccccc}
\hline \multirow{2}{*}{ ALUMNO } & \multicolumn{2}{c}{ ICV } & \multicolumn{2}{c}{ IRP } & \multicolumn{2}{c}{ IMT } & \multicolumn{2}{c}{ IVP } & \multicolumn{2}{c}{ CIT } \\
\cline { 2 - 11 } & Pre & Post & Pre & Post & Pre & Post & Pre & Post & Pre & Post \\
\hline DG & 102 & $\mathbf{1 4 6}$ & 119 & $\mathbf{1 2 1}$ & 110 & $\mathbf{1 1 0}$ & 112 & $\mathbf{1 1 8}$ & 114 & $\mathbf{1 3 2}$ \\
\hline JI & 124 & $\mathbf{1 3 8}$ & 104 & $\mathbf{1 2 5}$ & 102 & 97 & 94 & $\mathbf{1 0 3}$ & 109 & $\mathbf{1 2 3}$ \\
\hline HR & 100 & $\mathbf{1 3 4}$ & 110 & 106 & 94 & $\mathbf{9 7}$ & 100 & $\mathbf{1 1 5}$ & 103 & $\mathbf{1 1 9}$ \\
\hline DL & 95 & $\mathbf{1 5 0}$ & 106 & $\mathbf{1 2 5}$ & 80 & $\mathbf{9 4}$ & 100 & $\mathbf{1 0 6}$ & 94 & $\mathbf{1 2 8}$ \\
\hline KB & 77 & $\mathbf{1 4 8}$ & 94 & $\mathbf{1 1 7}$ & 104 & $\mathbf{1 1 0}$ & 97 & $\mathbf{1 1 2}$ & 89 & $\mathbf{1 3 0}$ \\
\hline SJ & 69 & $\mathbf{1 3 6}$ & 94 & $\mathbf{1 1 2}$ & 97 & $\mathbf{1 1 0}$ & 83 & $\mathbf{1 0 3}$ & 81 & $\mathbf{1 2 2}$ \\
\hline
\end{tabular}

Nota: Elaboración propia. Los números resaltados en negrita son aquellos que elevaron su puntuación en la etapa Post- test. ICV: Índice de Comprensión Verbal. IRP: Índice de Razonamiento Perceptual. IMT: Índice de Memoria de Trabajo. IVP: Índice de Velocidad de Procesamiento. CIT: Coeficiente Intelectual Total.

En el Pre-test, dos niños se clasificaron con una puntuación por debajo del promedio (KB y SJ), tres se situaron dentro del promedio (JI, HR y DL) y solo uno por encima del promedio (DG). Fue interesante reconocer que al finalizar el Proyecto Meccano la inteligencia de los estudiantes incrementó: un niño obtuvo un puntaje por encima del promedio (HR), tres se encontraron en el rango superior (JI, DL y SJ) y dos en el muy superior (DG y KB). Lo que sugiere que las capacidades cognitivas de los alumnos, con aptitud sobresaliente, incrementaron después de haber participado en las actividades del proyecto.

\section{Discusión}

Benavides et al. (2004) señalaron que, si bien se ha intentado garantizar la educación para todos los niños, aún existen grupos vulnerables que no reciben una atención acorde con sus necesidades. La atención educativa, para todas las personas independientemente de sus características, es un derecho que se ha promovido en el ámbito mundial, pero en ocasiones es difícil de lograr, debido a que los sistemas escolares no cuentan con la infraestructura conveniente para realizarlo. Es indudable que en el ámbito educativo falta mucho por hacer, pero 
si se comparan las condiciones de hace dos siglos con las actuales, se observa que los cambios establecidos en las políticas contribuyeron a tener mejores condiciones de vida. Por ejemplo, con la creación de la educación especial, se dio un paso importante para atender a los niños que, por diversas razones, no obtienen un aprendizaje satisfactorio, de acuerdo con lo establecido en el currículo escolar (Williams, 2001).

La Secretaría de Educación Pública -SEP- (2012) indicó que el enriquecimiento escolar se diseñó con el propósito de ofrecer alternativas adicionales para que los docentes diversifiquen la enseñanza y ofrezcan otras posibilidades para los alumnos con necesidades educativas. Por tanto, es una herramienta pedagógica que atiende la diversidad de los niños en el aula, con acciones para lograr el máximo aprendizaje, y desarrollo de competencias y habilidades, a través de la calidad y la equidad. De ahí que, Proyecto Meccano se creó bajo el sustento de favorecer las aptitudes sobresalientes de niños de primaria.

El primer paso fue la identificación de los estudiantes, que se realizó a partir de una propuesta multidimensional, con la que se reconoció a 15 estudiantes con aptitud sobresaliente (18\%); este dato es interesante y se acerca más a lo reportado en otros estudios, los cuales encontraron una incidencia que oscila entre un $3 \%$ y un $20 \%$ de esta población, a nivel primaria (Chávez y Acle, 2018; Gargiulo, 2012; Kelemen, 2012; Renzulli, 2011). Así mismo, como señaló Renzulli (2011), las principales características de las personas ubicadas en esta categoría son la interacción de tres rasgos humanos: coeficiente intelectual, compromiso con la tarea y creatividad.

Cabe mencionar que en este estudio participaron seis niños, quienes tuvieron el permiso de sus padres. Los alumnos se caracterizaron por presentar una elevada creatividad, razonamiento perceptual, motivación y un alto autoconcepto, pero se debían favorecer habilidades cognitivas como la observación, comparación, relación y clasificación. Lo anterior, sugiere que existen distintos perfiles y que el empleo de modelos multidimensionales es una buena alternativa para la detección de sus capacidades (Chávez y Acle, 2018; Covarrubias, 2018; Renzulli, 2011).

Con base en el perfil de los alumnos sobresalientes se diseñó el programa Meccano, para favorecer las habilidades básicas del pensamiento; este demostró que, a lo largo de su instrumentación, los niños mejoraron su capacidad para encontrar detalles, reconocer variaciones de tamaño, forma, cantidad, dirección de los objetos, figuras escondidas, secuencias numéricas; se apropiaron de sistemas de referencia, lograron comparar imágenes por medio de parámetros establecidos y buscaron información para clasificar objetos. 
La evidencia empírica de este trabajo permitió reconocer que los alumnos mejoraron sus capacidades cognoscitivas básicas, lo cual se sustenta con lo mencionado por Bello (2013), Duarte (2001), Velarde (2008), Howie (2015), Jiménez (2013), Mateus (2017), Ushiñahua y Vílchez (2016), quienes han encontrado que los programas de enriquecimiento promueven el funcionamiento cognitivo, ya que en el caso de los alumnos con aptitud sobresalientes se fortalece la profundidad de su aprendizaje, al proporcionar nuevas oportunidades y retos innovadores, adicionales a los establecidos en el currículo (Sánchez, 2003). De igual manera, concuerda con lo reportado por Booij et al. (2016), Chan et al. (2020), Chen y Chen (2020), García-Perales y Almeida (2019), Golle et al. (2019), Howie (2015), Mihyeon (2016), y Varela et al. (2006), al referir efectos positivos en las habilidades cognoscitivas de los niños, después de participar en programas de enriquecimiento, al favorecer la discriminación, raciocinio secuencial, establecimiento de relaciones entre las partes y el todo, generación de nueva información, a través de síntesis, y mayor eficiencia de pensamiento analógico.

Lo anterior, se corroboró en la etapa Post-test en donde se encontró que los participantes incrementaron su flexibilidad de pensamiento, sus ideas fueron originales, mejoraron su comprensión verbal, razonamiento perceptual, velocidad de procesamiento, memoria, motivación y su autopercepción como estudiantes. Lo anterior, concuerda con otros estudios que han observado beneficios en las capacidades de los estudiantes que participaron en programas de enriquecimiento (Bahar \& Ozturk, 2018; Chávez, 2008; Chávez et al., 2009; Howie, 2015; Klimenko y Botero, 2016). En este sentido, cobra relevancia lo señalado por Sternberg (1998, 2005), Golle et al. (2019), Howie (2015), Ushiñahua y Vílchez (2016), quienes mencionaron que la adquisición y consolidación de un conjunto de destrezas, permite el dominio de distintas áreas académicas y favorece el aprendizaje.

Por otro lado, se observó que en las variables afectivo-motivacionales el $80 \%$ de los alumnos fue capaz de reconocer sus capacidades como estudiantes y se percibieron como buenos en sus materias escolares. En este sentido, es importante considerar que el autoconcepto académico actúa como moderador del logro intelectual, favorece el desarrollo del potencial y éxito en el aprendizaje (Chávez, 2014; Hérbert \& Smith, 2018). Por lo que Mönks (1999), Preckel et al. (2008) y Renzulli (2011) mencionaron que esta variable actúa como predictor del rendimiento académico, y se debe considerar en el proceso de identificación de los alumnos sobresalientes que viven en contextos socioeconómicos vulnerables. En cuanto a la motivación intrínseca, evaluada a través del compromiso con la tarea, $50 \%$ de los niños indicó estar interesado por aprender sus materias escolares, esforzase y ser persistentes en sus actividades educativas. Al respecto, Agaliotis \& Kalyva (2019), Moritz et al. (2009), y Schick \& Phillipson (2009) encontraron en sus estudios que esta variable es una característica importante en esta 
población, logrando que los niños se distingan por ser perseverantes, lo cual se observa en la intensidad y devoción que dedican en las actividades que les agradan.

Gracias a esta investigación, fue posible encontrar, tal como lo comentó Carvalho (2013), que los programas de enriquecimiento son alternativas pedagógicas para estimular las habilidades cognoscitivas de los estudiantes con necesidades educativas especiales.

\section{Conclusiones}

Es claro que se han dado avances, principalmente en la creación de las políticas que han contribuido a que se tengan mejores condiciones de vida, pero aún falta mucho por hacer.

Al finalizar la instrumentación del programa de enriquecimiento se observaron distintos beneficios en los participantes; se encontró que ellos son capaces de seleccionar, organizar información relevante, y distinguir entre diferentes puntos de vista. Así mismo, mejoraron la interacción, comunicación, capacidad de reflexión, toma de decisiones y autocontrol.

La preeminencia de "Proyecto Meccano", es que las actividades que se realizaron son sencillas, fáciles de hacer, con instrucciones claras, pero al mismo tiempo complejas, al requerir que el alumno salga de la zona de confort e instarle a que vaya más allá de lo común, que piense en más de una solución, trabaje de manera individual y colaborativa.

Es necesario fortalecer las capacidades cognoscitivas, motivacionales y de personalidad de los alumnos con aptitud sobresaliente, para reafirmar sus fortalezas; por otra parte, prestar atención a los aspectos por potenciar, con el propósito de obtener un desarrollo apropiado en distintas áreas de su vida, ya que como se ha señalado en previas investigaciones, la adquisición y consolidación de un conjunto de destrezas permite el dominio de distintas áreas académicas y favorece el aprendizaje, lo cual a su vez influye a lo largo del ciclo de la vida de las personas.

Finalmente, la evidencia empírica de este trabajo permitió reconocer que los alumnos mejoraron sus capacidades cognoscitivas básicas, y que "Proyecto Meccano" es una alternativa viable para favorecer las necesidades educativas de los alumnos con aptitud sobresaliente. En este sentido, se destaca la importancia de continuar con el programa de enriquecimiento para trabajar y fortalecer otras habilidades del pensamiento. 
Enriquecimiento

de las habilidades
Enriquecimiento de las habilidades | Revista Virtual

Universidad Católica del Norte, 64, 65-91

ISSN: 0124-5821 (En línea)

\section{Referencias}

Agaliotis, I., \& Kalyva, E. (2019). Motivational differences of Greek gifted and Non gifted HighAchieving and Gifted under-achieving students. International Education Studies, 12(2), 45-56. https://doi.org/10.5539/ies.v12n2p45

Bahar, A., \& Ozturk, M. A. (2018). An exploratory study on the relationship between creativity and processing speed for gifted children. International Education Studies, 11(3), 77-91. https://doi.org/10.5539/ies.v11n3p77

Bello, J. (2013). "Viajeros del pensamiento": Enriquecimiento de las habilidades cognoscitivas básicas en alumnos sobresalientes (Tesis de maestría). Universidad Nacional Autónoma de México.

Benavides, M., Maz, A., Castro, E., y Blanco, R. (2004). La Educación de Niños con Talento en Iberoamérica. Trineo.

Booij, A., Haan, F., \& Plug, E. (2016). Enrichment Students Pays off: Evidence from an individualized gifted and talented program in secondary education. IZA Discussion Paper, (9757), 1-37. http://ftp.iza.org/dp9757.pdf

Carvalho, L. (2013). Programa de enriquecimiento instrumental una alternativa pedagógica para el desarrollo de habilidades y capacidades cognitivas en alumnos con necesidades educativas especiales. Universidad de Extremadura.

Chan, L. K., Chan, D., \& Sun, X. (2020). University-based gifted programs for gifted and talented students in Hong Kong: Practice and Evaluation. Gifted Education International, 2(36), 90-107. https://doi.org/10.1177/0261429420917858

Chávez, B. I. (2008). Programa de enriquecimiento para niños con potencial sobresaliente de segundo ciclo de primaria. (Tesis de Maestría). Universidad Nacional Autónoma de México.

Chávez, B. I. (2014). Evaluación Multidimensional de alumnos con aptitud sobresaliente de educación primaria (Tesis de doctorado). UNAM.

Chávez, B. I., y Acle, G. (2018). Niños con altas capacidades: análisis de las variables familiares implicadas en el desarrollo del potencial. Electronic Journal or Research in Educational psychology, 16(45), 273-300. http://dx.doi.org/10.25115/ejrep.v16i45.2094 
Enriquecimiento

de las habilidades
Enriquecimiento de las habilidades | Revista Virtual

Universidad Católica del Norte, 64, 65-91

ISSN: 0124-5821 (En línea)

Chávez, B. I., Zacatelco, F., y Acle, G. (2009). Programa de enriquecimiento de la creatividad para alumnas de zonas marinadas. Electronic Journal or Research in educational psychology, 7(2), 849-976. http://dx.doi.org/10.25115/ejrep.v7i18.1366

Chen, W. R., \& Chen, M-F. (2020). Practice and evaluation of enrichment programs for the gifted and talented learners. Gifted Education International, 2(36), 108-129. https://doi.org/10.1177/0261429420917878

Covarrubias, P. P. (2018). Del concepto de aptitudes sobresalientes al de altas capacidades y el talento. Revista de Investigación Educativa de la REDIECH, 9(7), 17-53. https://doi.org/10.33010/ie_rie_rediech.v9i17.123

Creswell, J. W., \& Plano, V. L. (2007). Designing and Conducting mixed method research. Sage.

De Pablo-Blanco, C., y Rodríguez, M. (2010). Manual práctico de discapacidad intelectual. Síntesis.

Duarte B. E. (2001). Modelo para la estimulación del pensamiento creativo (MEPC). McGrawHill.

Feuerstein, R. (1980). Instrumental enrichment. University Park Press.

Frola, R. P. (2004). Un Niño especial en mi aula. Trillas.

García-Perales, R., y Almeida, L. (2019). Programa de enriquecimiento para el alumnado con alta capacidad: Efectos positivos para el currículum. Revista Científica de Educomunicación, 60(27), 39-48. https://doi.org/10.3916/C60-2019-04

Gargiulo, R. (2012). Special education in contemporary society: an introduction to exceptionality. SAGE Publications.

Golle, J., Zettler, I., Rose, N., Trautwein, U., Hasselhorn, M., \& Nagengast, B. (2019). Effectiveness of a "Grass Roots" Statewide Enrichment Program for Gifted Elementary School Children. Journal of Research on Educational Effectiveness, 11(3), 375-408. https://doi.org/10.1080/19345747.2017.1402396

Hérber, T., \& Smith, K. (2018). Social and emotional development of gifted students. Gifted Child Today, 4(4), 176-186. https://doi.org/10.1177/1076217515597272

Hernández, I., Alvarado, J. C., y Luna, S. M. (2015). Creatividad e innovación: competencias genéricas o transversales en la formación profesional. Revista Virtual Universidad 
Enriquecimiento

de las habilidades
Enriquecimiento de las habilidades | Revista Virtual

Universidad Católica del Norte, 64, 65-91

ISSN: 0124-5821 (En línea)

Católica del Norte, 135-151. https://revistavirtual.ucn.edu.co/index.php/RevistaUCN/article/download/620/1155

Howie, D. (2015). The Feuerstein approach in New Zealand: Building on the past, for the future. Kairanga, 12(1), 37-44. https://files.eric.ed.gov/fulltext/EJ1240552.pdf

Jiménez, G. Y. (2013). Las Aventuras de Zarek Promoción de las capacidades analíticas, creativas y prácticas en alumnos sobresalientes (Tesis de maestría). Universidad Nacional Autónoma de México.

Kelemen, G. (2012). Identification of highly gifted children. Exedra, (6), 43-55. http://exedra.esec.pt/docs/N6/03-Edu.pdf

Kerlinger, F., y Lee, H. (2001). Investigación del Comportamiento. Métodos de investigación en Ciencias Sociales. McGraw-Hill.

King, S., y Cambell-Allan. (2006). Portafolio. Estrategia de reflexión, seguimiento, y evaluación del proceso de enseñanza-aprendizaje. Gaceta U.A.A., 8(3), 72.

Klimenko, O., y Botero, A. M. (2016). Fomento de la capacidad creativa desde las prácticas de enseñanza en una institución universitaria. Revista Psicoespacios, 10(17), 71-93. https://doi.org/10.25057/21452776.803

López, M. Á. (2008). Estudio, Mito y Realidad del niño sobredotado. Trillas

Marchesi, A., Coll, C., y Palacios, J. (2005). Desarrollo psicológico y educación. Alianza.

Mateus, Y. E. (2017). Efectividad de la aplicación de modificabilidad estructural cognitiva en la mejora del rendimiento académico de estudiantes de grado noveno. https://repository.ucatolica.edu.co/bitstream/10983/14057/3/ARTICULO.\%20terminado .pdf

Mihyeon, K. (2016). A Meta-Analysis of the Effects of Enrichment Programs on Gifted Students. Sage Journals, 60(2), 102-116. https://doi.org/10.1177/0016986216630607

Mönks, F. (1999). Desarrollo y educación de los niños superdotados. ¿Cómo pueden descubrir sus necesidades los padres y educadores? En Asociación Arangonesa de Psicopedagogía (Eds.), Respuestas Educativas para Alumnos Superdotados y Talentosos (pp. 173-186) Mira Editores. 
Enriquecimiento

de las habilidades
Enriquecimiento de las habilidades | Revista Virtual

Universidad Católica del Norte, 64, 65-91

ISSN: 0124-5821 (En línea)

Montoya, J. I., y Monsalve, J. C. (2008). Estrategias didácticas para fomentar el pensamiento crítico en el aula. Revista Virtual Universidad Católica del Norte, (25), 1-16. http://revistavirtual.ucn.edu.co/index.php/RevistaUCN/article/download/129/252

Moritz, K., Read, M., Clark, R., Callahan, C., \& Albaugh, S. (2009). Grade and gender differences in gifted students self-concepts. Journal for the Education of the Gifted, 32(3), 340-367. https://digitalcommons.unl.edu/edpsychpapers/125

Pérez, E., Furlan, L., Heredia, D., y Lescano, C. (2015). Estrategias, instrumentos y programas para la identificación y educación del talento. Anuario de Investigaciones de la $\begin{array}{llll}\text { Facultad } & \text { de } & \text { Psicología, } & \text { 2(1), }\end{array}$ https://revistas.unc.edu.ar/index.php/aifp/article/view/12860/13087

Preckel, F., Goetz, T. Pekrun, R. \& Kleine, M. (2008). Gender differences in gifted and average-

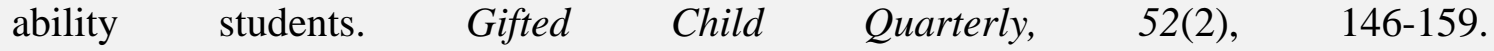
https://doi.org/10.1177/0016986208315834

Raven, J. C., Court, J. H., y Raven, J. (1993). Test de Matrices Progresivas Raven. Escala Coloreada, General y Avanzada. Paidós.

Renzulli, J. S. (2011). Theories, actions, and change: An academic journey in search of finding and developing high potential in young people. Gifted Child Quarterly, 55, 305-308. https://doi.org/10.1177/0016986211421875

Romero, S., y García, I. (2013). Educación especial en México. Desafíos de la educación inclusiva. Revista Latinoamericana de Educación Inclusiva, 7(2), 77-91. https://dialnet.unirioja.es/servlet/articulo?codigo $=4752911$

Rueda Romero, X. A. (2016). La comunicación de la ciencia y la tecnología como herramienta dialógica para la Apropiación Social de Ciencia, Tecnología e Innovación (ASCTeI) en comunidad Mixe, México. Trilogía Ciencia Tecnología Sociedad, 8(15), 119-128. https://doi.org/10.22430/21457778.407

Sánchez, M. E. (2003). Los niños superdotados: una aproximación a su realidad. Defensor del Menor en la Comunidad de Madrid. http://www.madrid.org/bvirtual/BVCM013887.pdf

Schick, H., \& Phillipson, S. (2009). Learmimg motivation and performance excellence in adolescents with high intellectual potential: what really matters? High Ability Studies, 20(1), 15-37. https://doi.org/10.1080/13598130902879366 
Enriquecimiento

de las habilidades
Enriquecimiento de las habilidades | Revista Virtual

Universidad Católica del Norte, 64, 65-91

ISSN: 0124-5821 (En línea)

Secretaría de Educación Pública -SEP-. (2006). Orientaciones generales para el funcionamiento de los servicios de educación especial. Secretaría de Educación Pública. http://www.educacionespecial.sep.gob.mx/pdf/publicaciones/libromorado.pdf

Secretaría de Educación Pública -SEP-. (2012). Educación inclusiva y recursos para la enseñanza. Estrategias específicas y diversificadas para la atención de alumnos y alumnas con discapacidad. Dirección de Educación Especial.

Sistema para el Desarrollo Integral de la Familia del Distrito Federal. (2014). Evaluación Interna 2014 del Programa de niñas y niños talento operado en 2012 -2013. http://intranet.dif.cdmx.gob.mx/transparencia/new/_evaluaciones/2014/Evaluacion\%20 Prog\%20Ninas\%20y\%20Ninos\%20Talento.pdf

Sternberg, R. J. (1998). A balance theory of wisdom. Review of General Psychology, 2(4), 347365. https://doi.org/10.1037/1089-2680.2.4.347

Sternberg, R. J. (2005). Augmenting the SAT through assessments of analytic, practical, and creative skills. En I. W. Kimmel (Ed.), Choosing students: Higher education admission tools for the 21st century (pp. 159-176). Earlbau.

Torrance, P. (2008). Research Review for the Torrance test of Creative Thinking Figural and Verbal Forms $A$ and $B$. Scholastic Testing Service. Inc.

Tzuriel, D., y Feuerstein, R. (1992). Dynamic assessment for prescriptive teaching. En C. Haywood y D. Tzuriel (Eds.), Interactive assessment, (pp. 187-206). Springer.

Unesco y Unicef. (2015). Declaración de Incheon educación 2030: Hacia una educación inclusiva y equitativa de calidad y un aprendizaje a lo largo de la vida para todos. http://portal.mec.gov.br/index.php?option=com_docman\&view= download\&alias=25981-spanish-version-ffa-and-ffa-and-incheon-declaration-1nov2015-pdf\&Itemid $=30192$

Ushiñahua, G. Y., y Vílchez, S. A. (2016). Programa basado en la modificabilidad estructural cognitiva y su influencia en el desarrollo cognitivo de estudiantes incluidos de educación primaria-Trujillo-2015. Ciencia y Tecnología, 12(3), 129-143. https://revistas.unitru.edu.pe/index.php/PGM/article/view/1520/1525

Varela, A., Gramacho, A., y Melo, C. (2006). Programa de enriquecimiento instrumental (PEI) alternativa pedagógica que responde al desafío de calidad educativa. Diversitas: 


\section{Enriquecimiento}

de las habilidades
Enriquecimiento de las habilidades | Revista Virtual Universidad Católica del Norte, 64, 65-91 ISSN: 0124-5821 (En línea)

$\begin{array}{lll}\text { Perspectivas } \quad \text { en } & \text { Psicología, } & \text { 2(2), }\end{array}$ https://www.redalyc.org/pdf/679/67920209.pdf

Velarde, E. (Julio-Diciembre, 2008). Teoría de la modificabilidad estructural cognitiva de Feuerstein. Investigación Educativa, 12(22), 203-221.

Wechsler, D. (2005). WISC-IV: La Escala Wechsler de Inteligencia para el Nivel Escolar. Manual Moderno.

Williams P. (2001). Diccionario enciclopédico de educación especial. Trillas.

Zacatelco, F. J. (2005). Modelo para la Identificación del niño sobresaliente en escuelas de educación primaria (Tesis de doctorado). Universidad Nacional Autónoma de México.

Zacatelco, F. J., Chávez, B. I., y González, A. (2013). Análisis Psicométrico de una escala de nominación del maestro para identificar aptitudes sobresalientes. XXXIV Congreso Internacional de Psicología.

Zacatelco, F. J., y Chávez, B. I. (2015). El enriquecimiento: Una alternativa educativa para estudiantes con aptitud sobresaliente. En F. Zacatelco (Eds.), Atención educativa para alumnos de primaria con aptitudes sobresalientes (pp. 105-132. Gedisa. 